\title{
Anxiety and depression in patients with pulmonary arterial hypertension and chronic thromboembolic pulmonary hypertension: Results from a Chinese survey
}

\author{
XIA ZHOU ${ }^{1}$, HUI SHI ${ }^{2}$, YUANHUA YANG ${ }^{3}$, ZUOQING ZHANG ${ }^{1}$, ZHENGUO ZHAI $^{4,5}$ and CHEN WANG ${ }^{4,5}$
}

${ }^{1}$ Department of Respiratory Medicine, Shijingshan Teaching Hospital of Capital Medical University, Beijing Shijingshan Hospital, Beijing 100043; Departments of ${ }^{2}$ Psychology and ${ }^{3}$ Respiratory Medicine,

Beijing Chao-Yang Hospital, Capital Medical University; ${ }^{4}$ Center of Respiratory Medicine, China-Japan Friendship Hospital, National Clinical Research Center for Respiratory Diseases, Peking University Health Science Center, Beijing 100029;

${ }^{5}$ Department of Respiratory Medicine, Capital Medical University, Beijing 100069, P.R. China

Received August 6, 2019; Accepted January 10, 2020

DOI: $10.3892 /$ etm.2020.8566

\begin{abstract}
Limited studies have focused on mental symptoms (anxiety and depression) and their impact on quality of life $(\mathrm{QoL})$ in patients with pulmonary hypertension $(\mathrm{PH})$. The objective of the present study was to assess the prevalence of anxiety and depression and their association with QoL in patients with pulmonary arterial hypertension (PAH) and chronic thromboembolic PH (CTEPH) in a Chinese population. Patients diagnosed with PH by right heart catheterization between March 2015 and February 2016 were recruited. QoL [short form 36 Health Survey Questionnaire (SF-36)] and depression (Patient Health Questionnaire depression scale-9) and anxiety (Generalized Anxiety Disorder-7) were assessed at baseline. Patients were followed up every 3 months. A total of 36 patients with PAH and 62 patients with CTEPH were enrolled. According to the results of the clinical examination and the questionnaires for depression and anxiety, patients were divided into two groups: Group 1, with depression and/or anxiety $(n=65,66.3 \%)$ and group 2 ,
\end{abstract}

Correspondence to: Professor Zhenguo Zhai, Center of Respiratory Medicine, China-Japan Friendship Hospital, National Clinical Research Center for Respiratory Diseases, Peking University Health Science Center, 2 East Yinghua Road, Chaoyang, Beijing 100029, P.R. China

E-mail: zhaizhenguo2011@126.com

Abbreviations: $\mathrm{PH}$, pulmonary hypertension; RHC, right heart catheterization; SF-36, short form 36 Health Survey Questionnaire; GAD-7, Generalized Anxiety Disorder-7; PHQ-9, Patient Health Questionnaire depression scale-9; QoL, quality of life; 6MWD, 6-minute walking distance; PCS, Physical Component Summary; MCS, Mental Component Summary; RP, role limitations due to physical health; BP, bodily pain; OR, odds ratio

Key words: pulmonary hypertension, depression, anxiety, survival, influencing factors without depression or anxiety $(n=33,33.7 \%)$. At baseline, the two groups did not differ in their hemodynamics. Patients with depression and/or anxiety (group 1) had a significantly lower score in all subscales of SF-36 $(\mathrm{P}<0.05)$. The impairment of QoL significantly correlated with the severity of depression $(\mathrm{P}<0.001)$ and anxiety $(\mathrm{P}<0.05)$. During the follow-up period, end-point events (death or admission due to deterioration) occurred in 17 patients $(17.3 \%) ; 8$ patients $(8.1 \%)$ were lost to follow-up. The end-point event-free survival rate in group 2 was significantly higher than that in group 1. Multivariate logistic regression analysis suggested that the financial situation and right ventricular enlargement were influencing factors of depression and/or anxiety. In conclusion, in patients with $\mathrm{PH}$ from a Chinese population, anxiety and depression were frequently encountered and significantly correlated with QoL. The significant relevant factors influencing anxiety/depression were the financial situation and right ventricular enlargement and patients with depression had a worse prognosis than those without.

\section{Introduction}

Pulmonary hypertension ( $\mathrm{PH})$ is defined as a disease or pathophysiological syndrome featured by increased pulmonary pressure: Mean pulmonary artery pressure $\geq 25 \mathrm{mmHg}$ measured by right heart catheterization (RHC) at rest (1). Usually, PH is insidiously progressive and clinically manifests as dyspnea, fatigue, chest pain, loss of weight, edema, weakness, palpitation and syncope, having a strong impact on patients' activities of daily life. The complex pathogenesis, limited treatment and no cure impose a psychological as well as economic burden on the patients.

According to pathology, hemodynamics, clinical diagnosis and treatment, PH is classified into five groups (1): Group I, pulmonary arterial hypertension (PAH); group II, $\mathrm{PH}$ due to left heart disease; group III, PH due to lung diseases and/or hypoxia; group IV, CTEPH and group V, PH with unclear multifactorial mechanisms. Evidence suggested that quality of life (QoL) is closely associated with age, gender, level of 
education, economic situation and supportive system (health care system and social society) (2,3). Symptoms, inflammatory cytokines, limitation of function, exercise capacity [6-minute walking distance (6MWD)], side effects of treatment, other chronic underlying diseases, the classification of $\mathrm{PH}$ and functional class of the patients were closely correlated with their health-associated QoL (2,4-7).

In addition, as with other chronic diseases, the uncertainty of treatment and limitation of activity in patients with $\mathrm{PH}$ frequently lead to anxiety and depression. One cross-sectional study indicated that $50.6 \%$ of the patients had depression, among which $19.2 \%$ of patients presented with severe depression, $14.8 \%$ had panic disorders and $7.1 \%$ had phobias. In patients with severe psychological problems, the QoL was obviously impaired (8). The reported mental disorders in patients with $\mathrm{PH}$ increased from $17.7 \%$ in New York Health Association (NYHA) class I to $61.9 \%$ in NYHA class IV (9). A study by Harzheim et al (10) revealed that scores in all aspects of SF-36 in patients with PH with severe mental disorders decreased and the severity of depression and anxiety had an apparent negative correlation with scores of QoL $(\mathrm{P}<0.05)$; in all dimensions of QoL, anxiety and depression appeared to be independent influencing factors (11).

With the improvement of modern medicine, the evaluation of prognosis should not only include objective parameters of disease but also include the comprehensive assessment of patients regarding their social status and psychological state. A previous study on PAH focused on survival and several biological parameters (12). To date, studies on the QoL, anxiety and depression in patients with PAH have been mostly cross-sectional; however, only few studies evaluated interventions regarding influencing factors and further observed the changes of symptoms, objective parameters, QoL and the mental condition after the intervention $(5-7,10,13)$. Domestic baseline data on depression, anxiety and QoL in patients with PH and further intervention are currently limited. Research on the QoL of patients with PAH and the relevant risk factors and the implementation of appropriate interventions are of vital clinical significance to improve patient prognosis.

While several studies demonstrated that medication improved the hemodynamics and exercise capacity of patients with $\mathrm{PH}$, their functional status and QoL was not improved accordingly $(4,13)$. QoL has been frequently ignored in clinical practice $(12,14,15)$, and to date, few studies $(2,5,9)$ have focused on mental symptoms (anxiety and depression) and their impact on QoL. Thus, the present study based on a Chinese population investigated anxiety and depression as well as QoL in patients with PH and discussed the following aspects: i) The association between mental symptoms (anxiety and depression) and QoL and the influencing factors; ii) the link between the presence of anxiety and/or depression and the outcome for patients with $\mathrm{PH}$, which provided a theoretical basis for preventing deterioration and improving survival.

\section{Patients and methods}

Study population. The present study was approved by the Ethics Committee of Beijing Chao-yang hospital (Beijing, China). Patients diagnosed with PH by RHC between March 2015 and February 2016 at Beijing Chao-yang Hospital (Beijing, China) were enrolled. All patients consented to study participation and provided written informed consent for the use and publication of their data in the present study. The inclusion criteria were as follows: i) Age $>18$ years and ii) the diagnosis was confirmed by RHC. The exclusion criteria were as follows: i) Patients had known mental diseases and intellectual disability recorded in their medical history; ii) cerebrovascular disease and senile dementia; iii) systematic hormonotherapy; iv) if patients had one of the following: Severe heart disease, dysfunction of kidney or liver or other organ dysfunction.

Collection of clinical baseline data. Patients meeting the inclusion criteria signed the informed consent form and their baseline data (age, sex, duration of symptoms, occupation, education, financial status) were collected, as well as their clinical data including $\mathrm{N}$-terminal pro-brain natriuretic peptide, blood gas analysis, pulmonary function test, cardiac color ultrasound, RHC and treatment. Questionnaires, including Generalized Anxiety Disorder-7 (GAD-7; none, score 0-4; mild, score 5-9; moderate, score 10-14; severe, 15-21), the Patient Health Questionnaire depression scale-9 (PHQ-9; none, score 0-4; mild, score 5-9; moderate, score 10-14; severe, score 15-19; very severe, score 20-27) and the short form 36 Health Survey Questionnaire (SF-36) (16) were applied to the patients.

Follow-up. Patients enrolled were followed up by telephone or at the outpatient department every 3 months to record whether an end-point event occurred. The total follow-up time was 1 year and the average follow-up time for the cohort was 6 months. The end-point event was defined as death or re-admission due to exacerbation. The definition of exacerbation included exacerbation of symptoms including cough, dyspnea, palpitation or syncope leading to admission or other conditions, including pneumonia requiring clinical treatment.

Statistical analysis. SPSS 18.0 software (SPSS, Inc.) was used to analyze the data. Descriptive statistics were used to indicate the characteristics of the participants. Continuous variables were expressed as the mean \pm standard deviation or median with range when appropriate. Enumeration data were expressed using the constituent ratio or rate. Continuous variables of the baseline characteristics were compared using analysis of variance. A linear correlation was assumed to assess the correlation between two variables. A Spearman's logistic regression analysis (univariate logistic regression analysis and multivariate logistic regression analysis) was performed to calculate odds ratios and $95 \%$ confidence intervals for different baseline characteristics regarding the impact on anxiety and/or depression was assessed. Variables with $\mathrm{P}<0.05$ in the univariate logistic regression analysis were subjected to multivariate logistic regression analysis. Follow-up results of patients were presented by Kaplan-Meier curves in a time-to-event model and compared between groups by using the log-rank test. All analyses were performed as two-sided significance tests and statistical significance was set at $\mathrm{P}<0.05$.

The SF-36 includes 36 items in 8 dimensions: Physical functioning, role limitations due to physical health (RP), body pain (BP), general health perceptions, vitality, social functioning, 
Table I. Comparison of baseline information.

\begin{tabular}{|c|c|c|c|}
\hline Parameter & Anxiety and/or depression $(n=65)$ & No anxiety or depression $(n=33)$ & P-value \\
\hline Age (years) & $47.94 \pm 12.84$ & $49.53 \pm 16.91$ & 0.606 \\
\hline Sex (male vs. female) & $20(30.8)$ & $18(54.5)$ & 0.023 \\
\hline $\mathrm{BMI}\left(\mathrm{kg} / \mathrm{m}^{2}\right)$ & $23.52 \pm 3.625$ & $23.55 \pm 3.183$ & 0.962 \\
\hline Course of disease (months) & $24(10-48)$ & $18(7-54)$ & 0.916 \\
\hline Classification of PH & & & 0.205 \\
\hline PAH (I) & $21(32.3)$ & $15(45.5)$ & \\
\hline CTEPH (IV) & $44(67.7)$ & $18(54.5)$ & \\
\hline Other underlying diseases & $28(43.1)$ & $9(27.1)$ & 0.122 \\
\hline CVP & $5(2.5-10)$ & $3(2-9)$ & 0.212 \\
\hline mPAP & $50.45 \pm 10.48$ & $55.33 \pm 16.22$ & 0.074 \\
\hline Cardiac index & $2.03(1.60-2.54)$ & $2.07(1.72-2.60)$ & 0.287 \\
\hline PVR & $1,097.15 \pm 543.49$ & $1,013.05 \pm 491.57$ & 0.472 \\
\hline 6MWD & $364.89 \pm 109.24$ & $403.91 \pm 87.41$ & 0.080 \\
\hline Score of Borg $\geq 4$ & $24(40)$ & $5(15.1)$ & 0.021 \\
\hline Cardiac function WHO III/IV & $34(52.3)$ & $10(30.3)$ & 0.036 \\
\hline FEV1/pre & $86.25 \pm 13.40$ & $75.04 \pm 14.77$ & 0.001 \\
\hline DLCO/VA & $75.93 \pm 19.56$ & $80.86 \pm 17.70$ & 0.287 \\
\hline NT-proBNP & $962.7(211.5-1,946.0)$ & $414.65(79.97-2,469.8)$ & 0.339 \\
\hline Major treatment & & & 0.743 \\
\hline Basic treatment & $16(24.6)$ & $6(18.2)$ & \\
\hline Surgery & $14(21.5)$ & $7(21.2)$ & \\
\hline Targeted medicine & $35(53.8)$ & $20(60.6)$ & \\
\hline Adverse event during follow-up & $15(23.1)$ & $2(6)$ & 0.022 \\
\hline
\end{tabular}

Values are expressed as $\mathrm{n}(\%)$, median (interquartile range) or the mean \pm standard deviation. Increased NT-proBNP was defined as $>300 \mathrm{pg} / \mathrm{ml}$. Targeted medicine: Ambrisentan, Bosentan, Sildenafil, Tadalafil and other approved drugs that belong to the class of endothelin receptor antagonists, Phosphodiesterase inhibitors-V or prostacyclin analogues. BMI, body mass index; CVP, central venous pressure; PVR, pulmonary vascular resistance; 6MWD, 6-minute walking distance; FEV1/pre, predicted forced expiratory volume in 1 sec; NT-proBNP; N-terminal pro-brain natriuretic peptide; $\mathrm{PH}$, pulmonary hypertension; $\mathrm{PAH}$, pulmonary arterial hypertension; CTEPH, chronic thromboembolic PH; mPAP, mean pulmonary artery pressure; WHO, World Health Organization; DLCO/VA, carbon monoxide diffusing capacity/alveolar volume.

role limitation due to emotional problems and mental health. The GAD-7 and PHQ-9 include 7 and 9 questions and aim to screen anxiety and depression, respectively $(17,18)$.

\section{Results}

Basic information of the participants. A total of 156 patients with $\mathrm{PH}$ were recruited for the present study. Of these, 40 cases were excluded, as RHC was not performed or the RHC data were not available. Furthermore, 1 patient was excluded for refusing to complete the SF-36, and 2 patients were excluded due to lack of the PHQ-9 and GAD-7 results. In addition, 15 patients were excluded due to undesired classifications of PH after further examination. Finally, 98 patients were enrolled.

The cohort of the present study included 36 patients with PAH and 62 patients with CTEPH. The number of patients with anxiety was $51(52 \%)$ and the number of patients with depression was $56(57.1 \%)$. Based on the results of the PHQ-9 and GAD-7 questionnaires, the patients were divided into two groups: Group 1 included patients with $\mathrm{PH}$ with anxiety and/or depression, while group 2 included patients with $\mathrm{PH}$ with no anxiety/depression. Group 1 comprised 65 patients (20 males and 45 females; average age, $47.94 \pm 12.84$ years). Group 2 consisted of 33 patients with PH (18 males, 15 females; average age, $49.53 \pm 16.91$ years), thus, $66.33 \%$ of the participants with $\mathrm{PH}$ had anxiety and/or depression, while less than half classified as 'no anxiety or depression' based on GAD-7 and PHQ-9. The two groups were mostly made up of patients with CTEPH: There were $44(67.7 \%)$ patients with CTEPH in group 1 and 18 $(54.5 \%)$ patients with CTEPH in group 2. In group 1, 16 patients $(24.6 \%)$ received basic treatment (Warfarin or conservative treatment), 14 patients $(21.5 \%)$ received pulmonary thrombendarterectomy during the follow-up period and 35 (53.8\%) received oral targeted drugs (endothelin receptor antagonist, phosphodiesterase inhibitors-V, prostacyclin analogues). In group 1, 6 patients $(18.2 \%)$ received basic treatment (Warfarin or etiological treatment), 7 patients $(21.2 \%)$ received pulmonary thrombendarterectomy during the follow-up period and $20(60.6 \%)$ received oral targeted drugs (endothelin receptor antagonist, phosphodiesterase inhibitors-V, prostacyclin analogues). A summary and comparison of demographics, functional classification, hemodynamics and major treatments is provided in Table I. 

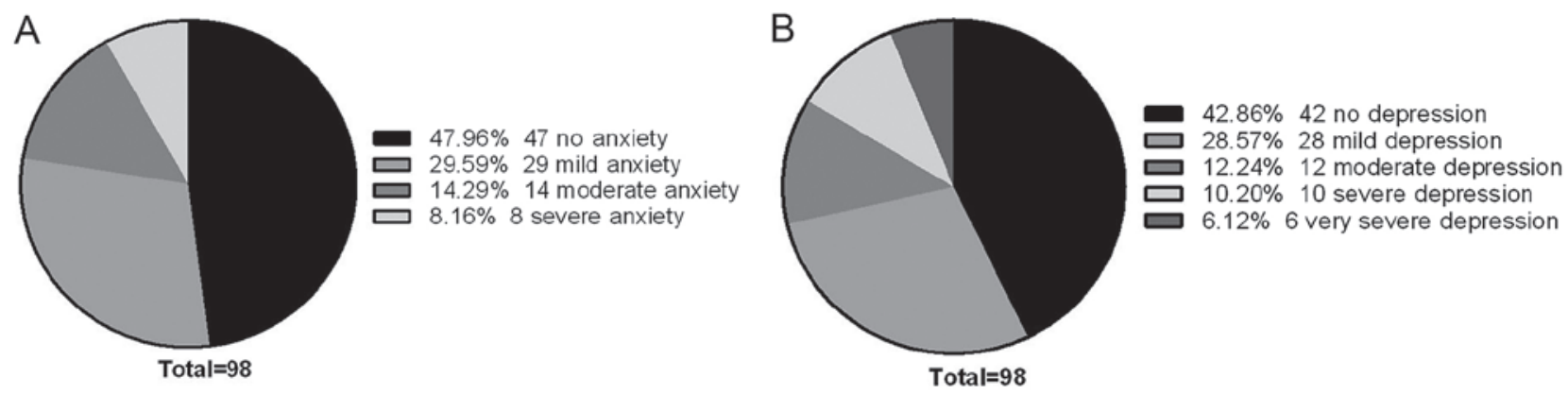

Figure 1. Pie charts presenting different degrees of anxiety and depression in the cohort. (A) The rate of anxiety in patients with PH was 52\%; (B) the rate of depression in patients with PH was 57 and $16 \%$ had severe/very severe depression. PH, pulmonary hypertension.
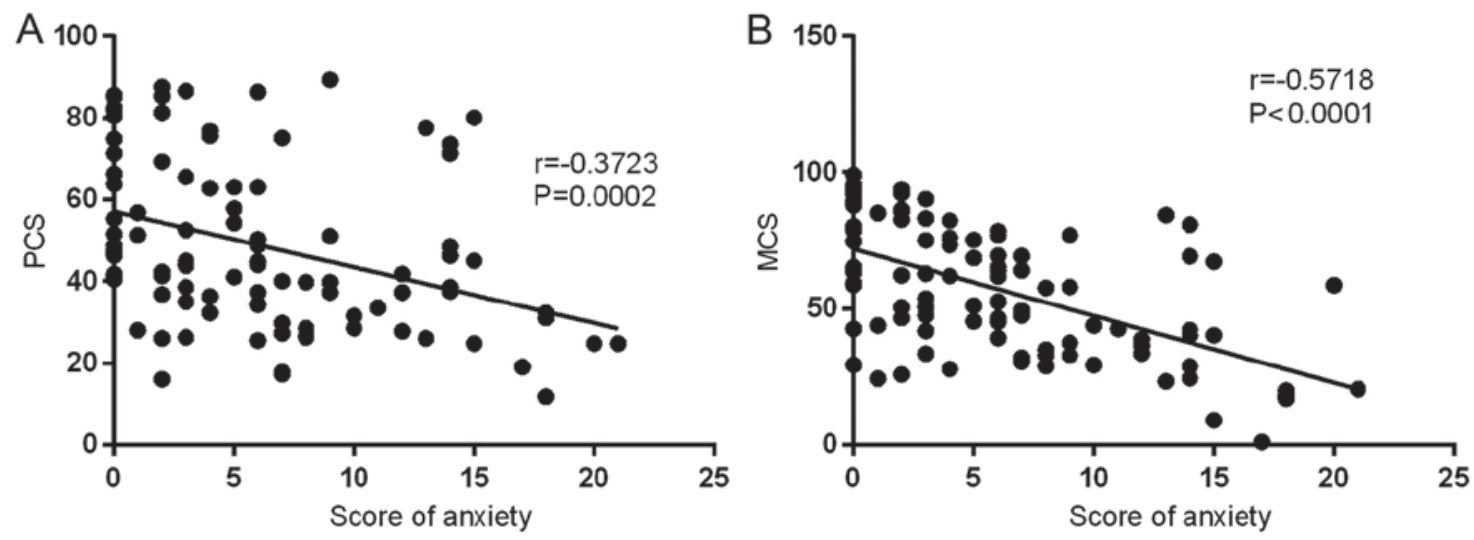

Figure 2. Scatter diagram and correlation analysis between anxiety and quality of life. Anxiety was negatively correlated with (A) PCS ( $\mathrm{r}=-0.3723, \mathrm{P}=0.0002)$ and (B) MCS (r=-0.5718, P<0.0001). PCS, Physical Component Summary; MCS, Mental Component Summary.

There was no significant difference between groups 1 and 2 for all patients with $\mathrm{PH}$, in the classification of $\mathrm{PH}, 6 \mathrm{MWD}$ and major treatment. However, the gender distribution, Borg scores and cardiac function classification were significantly different $(\mathrm{P}<0.05)$ : In the anxiety and/or depression group, the proportion of females and the Borg scores were higher than those group 2 (sex, $\mathrm{P}=0.023$; Borg score, $\mathrm{P}=0.021$ ); in addition, cardiac insufficiency was more severe in the anxiety and/or depression group $(\mathrm{P}=0.044)$.

In the study population, according to the GAD-7 and PHQ-9 score, respectively, 51 patients (52\%) had anxiety symptoms and the proportion of mild, moderate and severe anxiety was 30, 14 and $8 \%$, and 56 patients (57\%) had symptoms of depression. Depression was divided into four degrees: Mild depression (29\%), moderate depression (12\%), severe depression (10\%) and very severe depression (6\%; Fig. 1).

Correlation analysis between anxiety/depression and QoL. In SF-36, physical component summary (PCS) and mental component summary (MCS) were summarized. It was revealed that anxiety was negatively correlated with the Physical Component Summary (PCS) and was also negatively correlated with the Mental Component Summary (MCS; Fig. 2). The correlation analysis between depression and QoL indicated that depression was negatively correlated with PCS and MCS (Fig. 3).

Comparison of $Q o L$. In the anxiety and/or depression group, all dimensions of QoL were obviously more impaired than in the group without anxiety and/or depression and the difference was statistically significant $(R P, P=0.033 ; B P, P=0.016$; all other dimensions, $\mathrm{P}<0.01$; Fig. 4).

Logistic regression analysis of influencing factors of anxiety and/or depression. Based on the results of the univariate logistic-regression analysis (Table II), the multivariate logistic regression analysis was performed to identify influencing factors of anxiety and/or depression in patients with $\mathrm{PH}$ (Table III). The significant influencing factors were financial situation (OR, 4.616; 95\%CI, 1.498-14.227; $\mathrm{P}=0.008)$ and right ventricular enlargement (OR, 6.639; 95\%CI, 1.047-42.107; $\mathrm{P}=0.045$ ).

Results of end-point events and anxiety and/or depression. During the follow-up period, end-point events (death or admission due to deterioration) occurred in two cases (6\%) in group 2; by contrast, the number of cases with end-point events in group 1 was significantly higher $(n=15,23.1 \%, P=0.022$; Table I). Kaplan-Meier analysis indicated that survival without adverse events in group 2 was higher than that in group $1(\mathrm{P}=0.0730$; Fig. 5A). In the analysis for anxiety and depression individually, survival without adverse events in the patients with anxiety was lower than that in the patients without anxiety, but the difference was not significant ( $\mathrm{P}=0.3477$; Fig. 5B); however, survival without adverse event in patients with depression was significantly lower than that of the patients without depression ( $\mathrm{P}=0.008$; Fig. 5C). 

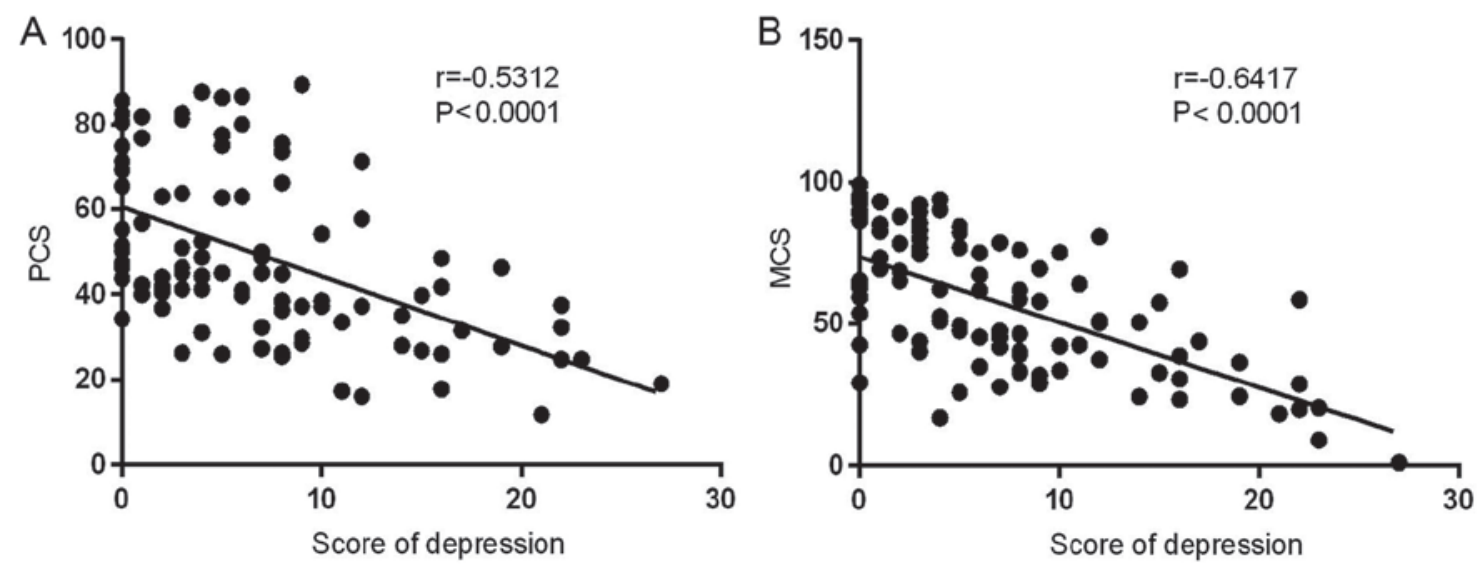

Figure 3. Scatter diagram and correlation analysis between depression and quality of life. Depression was negatively correlated with (A) PCS ( $\mathrm{r}=-0.5312$, $\mathrm{P}<0.0001)$ and (B) MCS ( $\mathrm{r}=-0.6417, \mathrm{P}<0.0001)$. PCS, Physical Component Summary; MCS, Mental Component Summary.

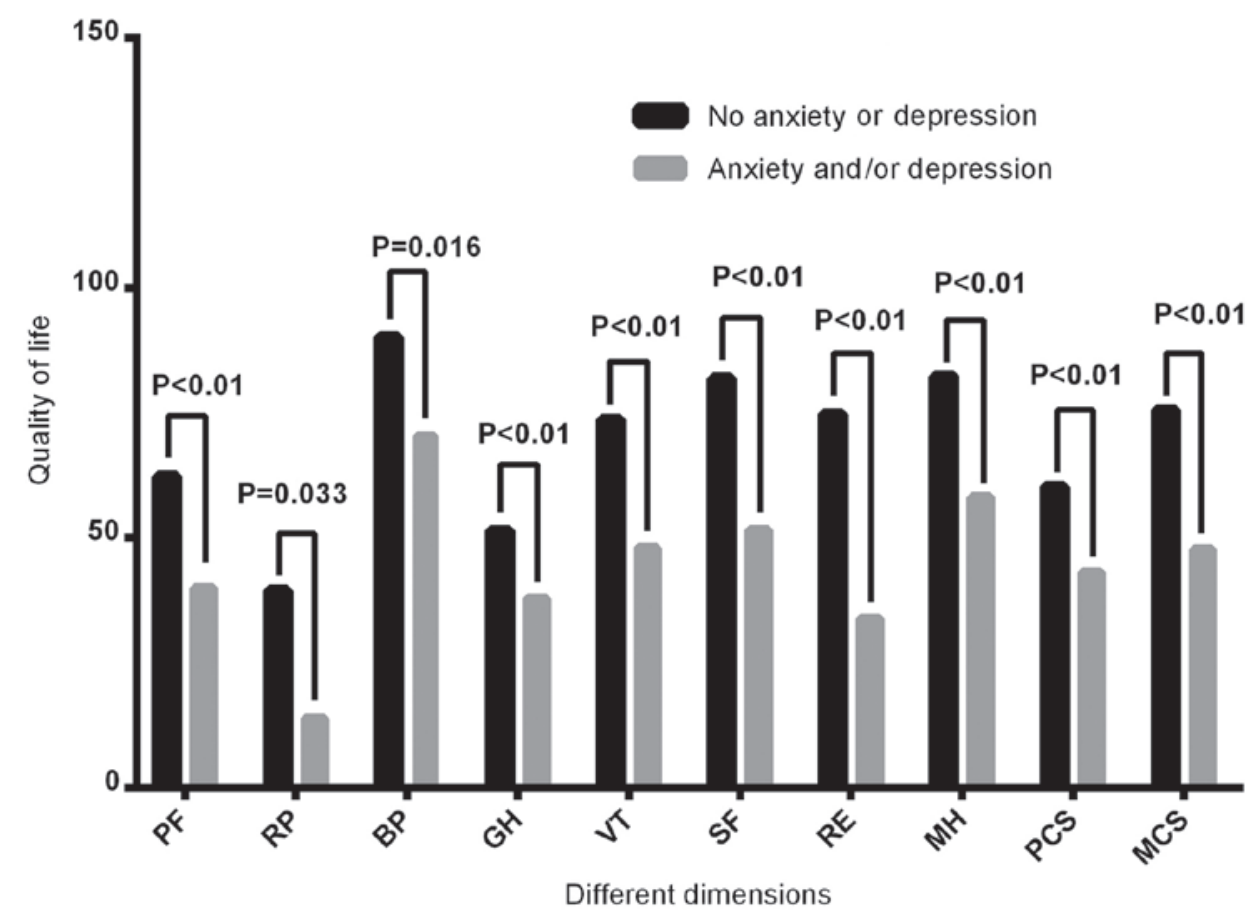

Figure 4. Comparison of quality of life between two groups in eight dimensions: PF, RP, BP, GH, VT, SF, RE, MH, PCS and MCS. PF, physical functioning; $\mathrm{RP}$, role limitations due to physical health; BP, bodily pain; GH, general health perceptions; VT, vitality; SF, social functioning; RE, role limitation due to emotional problems; MH, mental health; PCS, Physical Component Summary; MCS, Mental Component Summary.

\section{Discussion}

In the present study, 98 patients diagnosed with $\mathrm{PAH}$ or CTEPH by RHC were enrolled. The results of the questionnaires suggested that the incidence of anxiety and/or depression in patients with $\mathrm{PH}$ was considerable in the study population. In the present study, 51 patients (52\%) had anxiety, among which $22 \%$ had moderate and severe anxiety; furthermore, 56 patients (57\%) had depression, with $28 \%$ of patients having moderate or severe depression. There was an obvious correlation between the state of anxiety, depression and the impairment of QoL. Furthermore, the presence of depression was correlated with poor prognosis of the patients. The result of the high incidence of mental symptoms in patients with PAH and CTEPH was in accordance with previous studies in other countries $(5,8,14)$. By analyzing the association between anxiety, depression, $\mathrm{QoL}$ and prognosis, the present study emphasized the importance of incorporating mental health into the management of patients with $\mathrm{PH}$.

The association between mental health and QoL has been studied in multiple diseases, including cystic fibrosis, diabetes mellitus and heart diseases (10). The occurrence of mental disorders has been linked to difficulties in daily activities; however, whether difficulty in daily activities was the result of mental diseases or the cause of mental diseases remains elusive. Due to the type and severity of $\mathrm{PH}$, affected patients are vulnerable to emotional problems, including anxiety, depression and panic attacks (19). In patients with PAH, the estimated incidence of moderate to severe depression is $20-50 \%(5,20,21)$. One cross-sectional study revealed that 
Table II. Univariate logistic regression analysis of influencing factors of anxiety and/or depression.

\begin{tabular}{lcccr}
\hline & & & & \multicolumn{2}{c}{$95 \%$ CI } \\
\cline { 3 - 4 } Variable & P-value & OR & Lower & Upper \\
\hline Sex (male vs. female) & 0.746 & 1.111 & 0.588 & 2.100 \\
Marital status (married vs. unmarried) & 0.002 & 1.406 & 1.132 & 1.747 \\
University degree or higher & 0.001 & 1.481 & 1.171 & 1.874 \\
Financial situation (monthly income <2000RMB) & 0.024 & 1.383 & 1.043 & 1.835 \\
Targeted medicine & 0.002 & 1.577 & 1.189 & 2.091 \\
Other underlying diseases & 0.003 & 3.111 & 1.468 & 6.593 \\
Decreasing TAPSE & 0.007 & 2.048 & 1.215 & 3.450 \\
Right ventricular enlargement & 0.005 & 1.929 & 1.222 & 3.044 \\
Decreased LVEF (<50\%) & 0.341 & 3.000 & 0.312 & 28.841 \\
Right atrial enlargement & 0.002 & 2.174 & 1.327 & 3.562 \\
Pericardial effusion & 0.069 & 2.600 & 0.927 & 7.293 \\
Cardiac function WHO III/IV & 0.000 & 1.686 & 1.265 & 2.248 \\
6MWD <350 meters & 0.003 & 3.571 & 1.545 & 8.257 \\
Obstructive ventilation function disturbance & 0.732 & 0.889 & 0.453 & 1.743 \\
Score of Borg $\geq 4$ & 0.001 & 4.800 & 1.831 & 12.58 \\
Increased NT-proBNP & $<0.001$ & 2.765 & 1.588 & 4.815 \\
\hline
\end{tabular}

OR, odds ratio; TAPSE, tricuspid annular plane systolic excursion; LVEF, left ventricular ejection fraction; 6MWD, 6-minute walking distance; WHO, World Health Organization; NT-proBNP, N-terminal pro-brain natriuretic peptide. Increased NT-proBNP was defined as $>300 \mathrm{pg} / \mathrm{ml}$.

Table III. Multivariate logistic regression analysis of influencing factors of anxiety and/or depression.

\begin{tabular}{lccrr}
\hline & & & & \multicolumn{2}{c}{$95 \%$ CI } \\
\cline { 3 - 5 } Variable & P-value & OR & Lower & Upper \\
\hline Marital status (married vs. unmarried) & 0.841 & 1.142 & 0.314 & 4.158 \\
University degree or higher & 0.722 & 1.246 & 0.372 & 4.170 \\
Financial situation (monthly income <2000RMB) & 0.008 & 4.616 & 1.498 & 14.227 \\
Targeted medicine & 0.614 & 0.769 & 0.278 & 2.132 \\
Other underlying diseases & 0.214 & 0.476 & 0.148 & 1.534 \\
Decreasing TAPSE & 0.218 & 2.500 & 0.582 & 10.737 \\
Right ventricular enlargement & 0.045 & 6.639 & 1.047 & 42.107 \\
Right atrial enlargement & 0.143 & 0.253 & 0.04 & 1.594 \\
Cardiac function WHO III/IV & 0.506 & 0.643 & 0.175 & 2.364 \\
6MWD <350 meters & 0.301 & 0.493 & 0.129 & 1.884 \\
Score of Borg $\geq 4$ & 0.432 & 0.587 & 0.155 & 2.220 \\
Increased NT-proBNP & 0.567 & 0.655 & 0.154 & 2.789 \\
\hline OR, odds ratio; TAPSE, tricuspid annular plane systolic excursion; 6MWD, 6-minute walking distance; WHO, World Health Organization; \\
NT-proBNP, N-terminal pro-brain natriuretic peptide.
\end{tabular}

$19.2 \%$ of patients with $\mathrm{PH}$ developed severe depression and may develop other types of mental disorders, yet only $23.4 \%$ of patients from outpatient department and $8.6 \%$ patients from the inpatient department were receiving psychotherapy, while more than half wished for a relevant therapy (8). Furthermore, $>90 \%$ of patients at the inpatient department with moderate to severe mental problems had not been treated with psychoactive medication (8). The present result that the incidence of anxiety and/or depression in patients with PAH and CTEPH was high was in accordance with those of previous studies (8-10).

The present study revealed that anxiety and depression were negatively correlated with PCS and MCS of QoL. The QoL score in all dimensions was decreased in patients with 

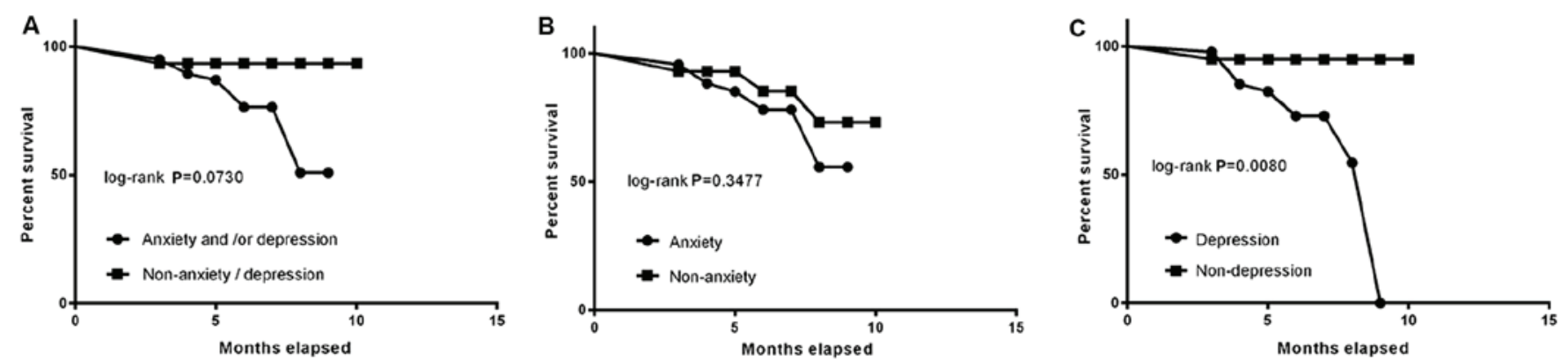

Figure 5. Kaplan-Meier analysis of survival without adverse events in patients with or without (A) anxiety/depression, (B) anxiety and (C) depression. The survival without adverse events in patients with depression was lower than that in the patients without depression $(\mathrm{P}=0.016)$.

PAH and CTEPH with anxiety and/or depression status compared with those without anxiety or depression.

In the present study, anxiety and/or depression occurred more frequently in females ( 75 vs. $53 \%$ in males), which was in accordance with previous results indicating that females were vulnerable to mental diseases (22). It has been demonstrated that anxiety and depression are common complications of chronic obstructive disease (23). The present study indicated that the significant influencing factors of anxiety/depression were the financial situation and right ventricular enlargement. The conclusion that the financial situation as a significant influencing factor of anxiety/depression was in accordance with a previous descriptive study by our group, according to which patients with PH reported that the financial burden due to their diseases was heavy and they had emotional problems (24). It appears that the self-assessment of the mental condition and QoL by patients, together with clinical parameters determined by clinicians, may be part of a comprehensive assessment.

Harzheim et al (10) indicated that although most patients did not receive psychological treatment, there was no difference in long-term survival between patients with moderate, severe, mild or no anxiety and depression. The rate of end-point events (death or hospitalization due to exacerbation) in the group with anxiety and/or depression was much higher than that in the non-anxiety/depression group during the follow-up period. The prognosis of patients with anxiety was inferior to that of the patients without anxiety, but the difference was not statistically significant. However, adverse events more frequently occurred in patients with depression than in patients without depression and the difference was statistically significant. Compared with patients with anxiety, patients with depression tend to develop adverse outcomes, suggesting that depression may be associated with constant reflection of the physical condition on the mental state and was more associated with prognosis.

Of note, the present study had certain limitations. It was difficult to perform a subgroup analysis for anxiety and/or depression and QoL in different types of patients with $\mathrm{PH}$ due to the small sample and relatively short follow-up period in the present study; thus, it is required to widen the sample size and prolong the follow-up period in a further study. As another limitation, screening tools for anxiety and depression were used, which identified symptoms but were not able to make a definite diagnosis for mental disease. The use of those tools may be associated with considerable bias. The patients were screened with self-assessment questionnaires. In the medical procedure, the patients' mental symptoms were frequently ignored. The present initial survey was made to call for more attention with this regard and more studies in China. A psychiatrist is required to make a definite diagnosis using more professional tools (Chinese version of the Structured Clinical Interview for Diagnostic and Statistical Manual of Mental Disorders, Fourth Edition $(25,26)$ and this may be performed in further studies by our group. Thus, cooperation with the psychological department is required for screening and further diagnosis in order to guide clinical management and specific treatment if required. Finally, the present study did not investigate whether the patients received psychological intervention and the outcome of anxiety and depression was not determined; therefore, the relevance between the outcome of anxiety and depression and the clinical outcome was not discussed in the present study. Future studies are required to answer those questions.

Regarding prospects for future research, previous studies to evaluate the impact of PH therapy on anxiety and depression were limited. One study concluded that the rate of anxiety and/or depression in patients with PH using Epoprostenol was lower than in those who did not (4). It was also reported that outcomes in patients with PAH using selective serotonin reuptake inhibitors (SSRIs) to treat depression may be better; however, due to multiple effects of SSRIs, this conclusion requires to be further confirmed (27). A later study indicated that SSRIs were associated with an increased death rate and clinical deterioration risk in PAH (28). Exercise training as an adjuvant therapy may improve exercise capacity, QoL and relative parameters of long-term outcome in patients with inoperable or residual CTEPH and may improve the 1, 2 and 3-year survival rate (15). It may be proposed that a similar adjuvant therapy may improve anxiety and depression in patients similar to those of the present cohort and further studies are required to provide evidence.

In conclusion, even though optimized treatments for $\mathrm{PH}$ are now available, anxiety and/or depression were frequently identified in such patients and were significantly correlated with impaired QoL. The present study based on a Chinese population calls for the necessity of including the screening for and diagnosis of anxiety and depression by professional questionnaires into routine clinical examination. In addition, there should be more randomized controlled trials to address the role of interventions including psychological consultation, 
psychological-social support and medication in patients with PH with anxiety and depression.

\section{Acknowledgements}

Not applicable.

\section{Funding}

The present study was supported by the fund of the National Natural Science Foundation of China (grant no. 81570049), the Beijing Natural Science Foundation (grant no. 7152062) and the National Key Research and Development Program of China (grant no. 2016YFC0905600).

\section{Availability of data and materials}

The datasets used and/or analyzed during the present study are available from the corresponding author on reasonable request.

\section{Authors' contributions}

$\mathrm{XZ}$, HS and ZhZ were responsible for patient data collection, analysis and interpretation, and were major contributors in writing the manuscript. $\mathrm{CW}, \mathrm{YY}$ and $\mathrm{ZuZ}$ made substantial contributions to the conception and design of the study and were involved in drafting the manuscript. All authors agreed to be accountable for all aspects of the work in ensuring that questions associated with the accuracy or integrity of any part of the work are appropriately investigated and resolved. All authors read and approved the final manuscript.

\section{Ethics approval and consent to participate}

This study was approved by the Ethics Committee of Beijing Chao-yang Hospital (Beijing, China). Written informed consent to participate was obtained from all patients and was in written form.

\section{Patient consent for publication}

Not applicable.

\section{Competing interests}

The authors declare that they have no competing interests.

\section{References}

1. Galiè N, Humbert M, Vachiery JL, Gibbs S, Lang I, Torbicki A, Simonneau G, Peacock A, Vonk Noordegraaf A, Beghetti M, et al: 2015 ESC/ERS guidelines for the diagnosis and treatment of pulmonary hypertension: The joint task force for the diagnosis and treatment of pulmonary hypertension of the European society of cardiology (ESC) and the European respiratory society (ERS) Endorsed by: Association for European paediatric and congenital cardiology (AEPC), international society for heart and lung transplantation (ISHLT). Eur Heart J 37: 67-119, 2016.

2. Matura LA, McDonough A and Carroll DL: Symptom prevalence, symptom severity, and health-related quality of life among young, middle, and older adults with pulmonary arterial hypertension. Am J Hosp Palliat Care 33: 214-221, 2016.
3. Heyworth IT, Hazell ML, Linehan MF and Frank TL: How do common chronic conditions affect health-related quality of life. Br J Gen Pract 59: e353-e358, 2009.

4. Shafazand S, Goldstein MK, Doyle RL, Hlatky MA and Gould MK: Health-related quality of life in patients with pulmonary arterial hypertension. Chest 126: 1452-1459, 2004.

5. Batal O, Khatib OF, Bair N, Aboussouan LS and Minai OA: Sleep quality, depression, and quality of life in patients with pulmonary hypertension. Lung 189: 141-149, 2011.

6. Rubenfire M, Lippo G, Bodini BD, Blasi F, Allegra L and Bossone E: Evaluating health-related quality of life, work ability, and disability in pulmonary arterial hypertension: An unmet need. Chest 136: 597-603, 2009.

7. Chua R, Keogh AM, Byth K and O'Loughlin A: Comparison and validation of three measures of quality of life in patients with pulmonary hypertension. Intern Med J 36: 705-710, 2006.

8. Larisch A, Neeb C, de Zwaan M, Pabst C, Tiede H, Ghofrani A, Olsson K, Hoeper M and Kruse J: Mental distress and wish for psychosomatic treatment of patients with pulmonary hypertension. Psychother Psychosom Med Psychol 64: 384-389, 2014 (In German).

9. Löwe B, Gräfe K, Ufer C, Kroenke K, Grünig E, Herzog W and Borst MM: Anxiety and depression in patients with pulmonary hypertension. Psychosom Med 66: 831-836, 2004.

10. Harzheim D, Klose H, Pinado FP, Ehlken N, Nagel C, Fischer C, Ghofrani A, Rosenkranz S, Seyfarth HJ, Halank M, et al: Anxiety and depression disorders in patients with pulmonary arterial hypertension and chronic thromboembolic pulmonary hypertension. Respir Res 14: 104, 2013.

11. Halank M, Einsle F, Lehman S, Bremer H, Ewert R, Wilkens H, Meyer FJ, Grünig E, Seyfarth HJ, Kolditz M, et al: Exercise capacity affects quality of life in patients with pulmonary hypertension. Lung 191: 337-343, 2013.

12. Flattery MP, Pinson JM, Savage L and Salyer J: Living with pulmonary artery hypertension: Patients' experiences. Heart Lung 34: 99-107, 2005.

13. Cicero C, Franchi SM, Barreto AC and Lopes AA: Lack of tight association between quality of life and exercise capacity in pulmonary arterial hypertension. Arq Bras Cardiol 99: 876-885, 2012 (In English, Portuguese).

14. McCabe C, Bennett M, Doughty N, MacKenzie Ross R, Sharples L and Pepke-Zaba J: Patient-reported outcomes assessed by the CAMPHOR questionnaire predict clinical deterioration in idiopathic pulmonary arterial hypertension and chronic thromboembolic pulmonary hypertension. Chest 144: 522-530, 2013.

15. Nagel C, Prange F, Guth S, Herb J, Ehlken N, Fischer C, Reichenberger F, Rosenkranz S, Seyfarth HJ, Mayer E, et al: Exercise training improves exercise capacity and quality of life in patients with inoperable or residual chronic thromboembolic pulmonary hypertension. PLoS One 7: e41603, 2012.

16. Li L, Wang HM and Shen Y. Chinese SF-36 health survey: Translation, cultural adaptation, validation, and normalisation. J Epidemiol Community Health 57: 259-263, 2003.

17. Spitzer RL, Kroenke K, Williams JB and Löwe B: A brief measure for assessing generalized anxiety disorder: The GAD-7. Arch Intern Med 166: 1092-1097, 2006.

18. Kroenke K, Spitzer RL and Williams JB: The PHQ-9: Validity of a brief depression severity measure. J Gen Intern Med 16: 606-613, 2001.

19. Matura LA, McDonough A and Carroll DL: Health-related quality of life and psychological states in patients with pulmonary arterial hypertension. J Cardiovasc Nurs 29: 178-184, 2014.

20. McCollister DH, Beutz M, McLaughlin V, Rumsfeld J, Masoudi FA, Tripputi M, Yaeger T, Weintraub P and Badesch DB: Depressive symptoms in pulmonary arterial hypertension: Prevalence and association with functional status. Psychosomatics 51: 339-339.e8, 2010.

21. White J, Hopkins RO, Glissmeyer EW, Kitterman N and Elliott CG: Cognitive, emotional, and quality of life outcomes in patients with pulmonary arterial hypertension. Respir Res 7: $55,2006$.

22. Buoli M, Caldiroli A, Serati M, Grassi S and Altamura AC: Sex steroids and major psychoses: Which role for DHEA-S and progesterone. Neuropsychobiology 73: 178-183, 2016.

23. Maurer J, Rebbapragada V, Borson S, Goldstein R, Kunik ME, Yohannes AM and Hanania NA; ACCP Workshop Panel on Anxiety and Depression in COPD: Anxiety and depression in COPD: Current understanding, unanswered questions, and research needs. Chest 134 (4 Suppl): 43S-56S, 2008. 
24. Zhai Z, Zhou X, Zhang S, Xie W, Wan J, Kuang T, Yang Y, Huang $\mathrm{H}$ and Wang $\mathrm{C}$ : The impact and financial burden of pulmonary arterial hypertension on patients and caregivers: Results from a national survey. Medicine (Baltimore) 96: e6783, 2017.

25. First MB, Spitzer RL, Gibbon M and Williams JBW: Structured clinical interview for DSM-IV-TR axis I disorders, research version, patient edition (SCID-I/P). New York: Biometrics Research, New York State Psychiatric Institute, 2002.

26. Kam IW: Development of the bilingual (Chinese/English) SCID-I (Structured Clinical Interview for DSM-IV axis I disorder): A study of its reliability and validity in an in-patient population (Dissertation for Part III Examination of FHKCPsych) Hong Kong College of Psychiatrist, Hong Kong, 2000.
27. Kawut SM, Horn EM, Berekashvili KK, Lederer DJ, Widlitz AC, Rosenzweig EB and Barst RJ: Selective serotonin reuptake inhibitor use and outcomes in pulmonary arterial hypertension. Pulm Pharmacol Ther 19: 370-374, 2006.

28. Sadoughi A, Roberts KE, Preston IR, Lai GP, McCollister DH, Farber HW and Hill NS: Use of selective serotonin reuptake inhibitors and outcomes in pulmonary arterial hypertension. Chest 144: 531-541, 2013. 